\title{
DESIGN, CONSTRUCTION AND IN SITU TESTING OF A MUON CAMERA FOR EARTH SCIENCE AND CIVIL ENGINEERING APPLICATIONS.
}

\author{
Ignacio Lázaro Roche ${ }^{1,2,3,4^{*}}$, Adnand Bitri $^{1}$, Simon Bouteille ${ }^{3}$, Jean-Baptiste Decitre ${ }^{4}$, Kevin \\ Jourde $^{1}$, Julien Gance ${ }^{3}$, Catherine Truffert ${ }^{3}$, and Stéphane Gaffet ${ }^{2,4}$ \\ ${ }^{1}$ BRGM; Orléans, France \\ ${ }^{2}$ Geoazur, Univ. of Nice, CNRS, IRD, OCA; Sophia-Antipolis, France \\ ${ }^{3}$ Iris Instruments; Orléans, France \\ ${ }^{4}$ LBNL, Aix-Marseille Univ., CNRS, Univ. of Avignon, Univ. of Nice; Rustrel, France
}

\begin{abstract}
The MUST ${ }^{2}$ (MUon Survey Tomography based on Micromegas detectors for Unreachable Sites Technology) camera is based on a thin Time Projection Chamber read by a resistive Micromegas. This innovative combination presents interesting distinctive features compared to existing muon detection technologies. It allows a wide angular acceptance of the detector with a low weight and compact volume, well adapted for confined spaces or underground operation. The current work presents the results obtained during the calibration measurements at the reference site, the Low Background Noise Laboratory (LBNL). Preliminary results from field measurement campaign carried out at the dam overlooking the village of Saint-Saturnin-les-Apt (South-East of France) are presented and discussed.
\end{abstract}

\section{Introduction}

Muography is a rising technique with promising applications. It was introduced in the late '70s by Alvarez and collaborators to unravel the secrets of the Egyptian pyramids [1]. Through the years, several devices allowed muon detection based on different physical principles of its interaction with matter. Nowadays there is a broad range of cosmic muon detection technologies with different assets depending on the targeted application.

Cosmic muons are naturally originated in the upper atmosphere with a set of properties that allow them to propagate up to several hundreds of meters underground. The muon flux decreases progressively with an absorption proportional to the medium thickness and density (i.e. opacity), setting the basis for transmission muography (TM). TM provides reliable, original and independent information relatively to the physics of the measures produced by the seismic, or resistivity soundings of the Earth for instance.

Used as a complementary method in several disciplines (e.g., volcanology, nuclear management or carbon capture and sequestration [2-4]), TM eases the resolution of the inverse problem and improves the resolution of the density distribution of big volumes.

* Corresponding author: ignacio.lazaro@1sbb.eu 
Regarding the present work, the Temporal Tomography of Densitometry by the measurement of muons (T2DM2) collaboration aims at developing a new non-destructive system using cosmic muons for in-situ imaging and temporal monitoring the density of large volumes of matter. This new geophysical tool is meant to image the time-space variations of the medium density in order to monitor civil engineering works, geological, mineral or hydrogeological volumes.

\section{Calibration}

The MUST ${ }^{2}$ camera is a gaseous muon detector conceived, both for ground- and underground operation, as a compact, portable and robust tool for geophysics and civil engineering. The detector consists of a thin time projection chamber (TPC) with a micromegas readout plane and the acquisition is triggered by the coincidence signal of two coupled scintillators. The angular acceptances of this configuration are $\pm 80^{\circ}$ of zenith and $360^{\circ}$ of azimuth angles in polar coordinates as seen in Figure 1. A detailed description of the technology, its functioning principles and the experimental setup can be found in [8].

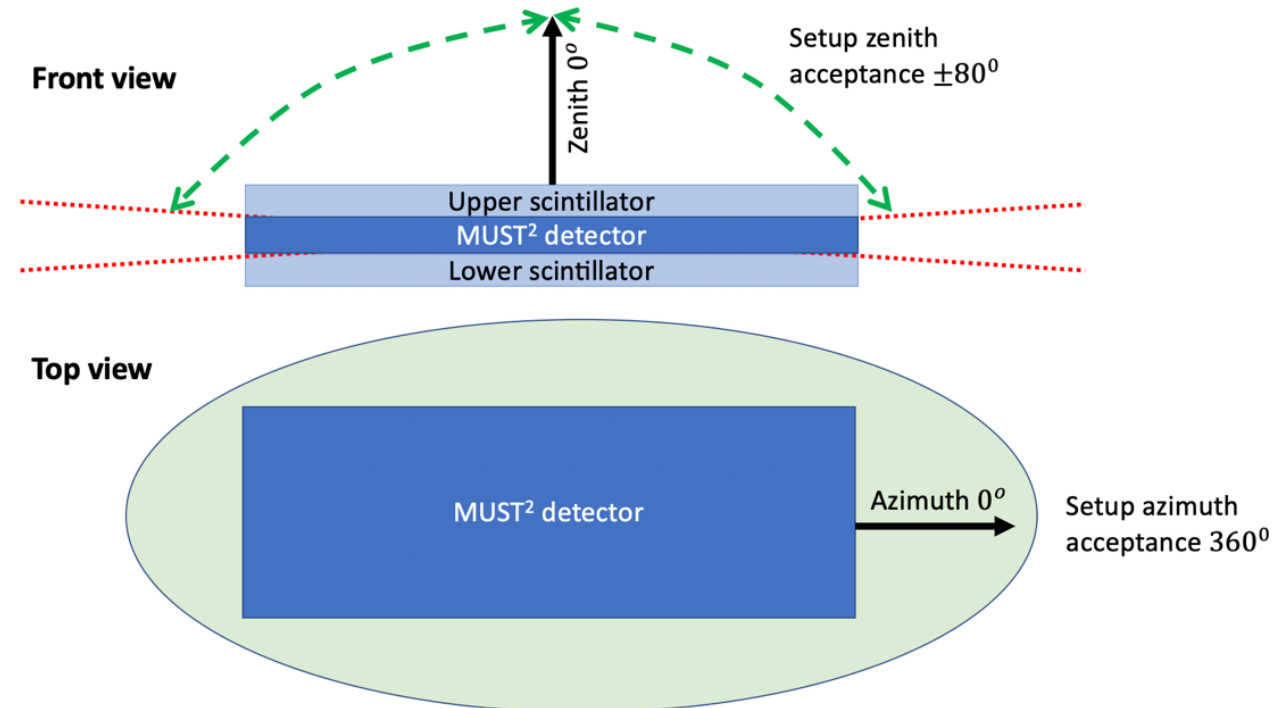

Fig. 1. Scheme of the calibration setup and angular acceptances.

To quantify the flux's drop for imaging purposes, it is necessary to get a precise muon flux characterization at ground level as a reference input for the tomography. The empirical model used during the present work is based on the Gaisser's model and improved by Tang and Lesparre [5-7]. It allows to estimate the theoretical muon's flux expected at sea level.

The first campaign of measurements aims at validating the track reconstruction efficiency by comparing the experimental results of an acquisition in open-sky conditions (no massive obstacles within the angular acceptance field of view of the detector) to the number of muons predicted by the theoretical model. This campaign has been carried out on the top of the mountain hosting the LBNL facilities, the Vestale site (latitude $43,942122^{\circ} \mathrm{N}$, longitude $5,48551^{\circ} \mathrm{E}, 1017 \mathrm{~m}$ above sea level).

The three charts of Figure 2 illustrate a polar chart of muon's arrival distribution. The red concentric lines represent the zenith angle $\left(\theta, 0^{\circ}=\right.$ zenith, $90^{\circ}=$ horizon $)$ and the black arrow the azimuth $\left(\varphi, \mathrm{Phi}=0^{\circ}\right.$ stands for the reference azimuth of the detector, during the presented acquisition was aligned to $63^{\circ}$ of geographic north). 

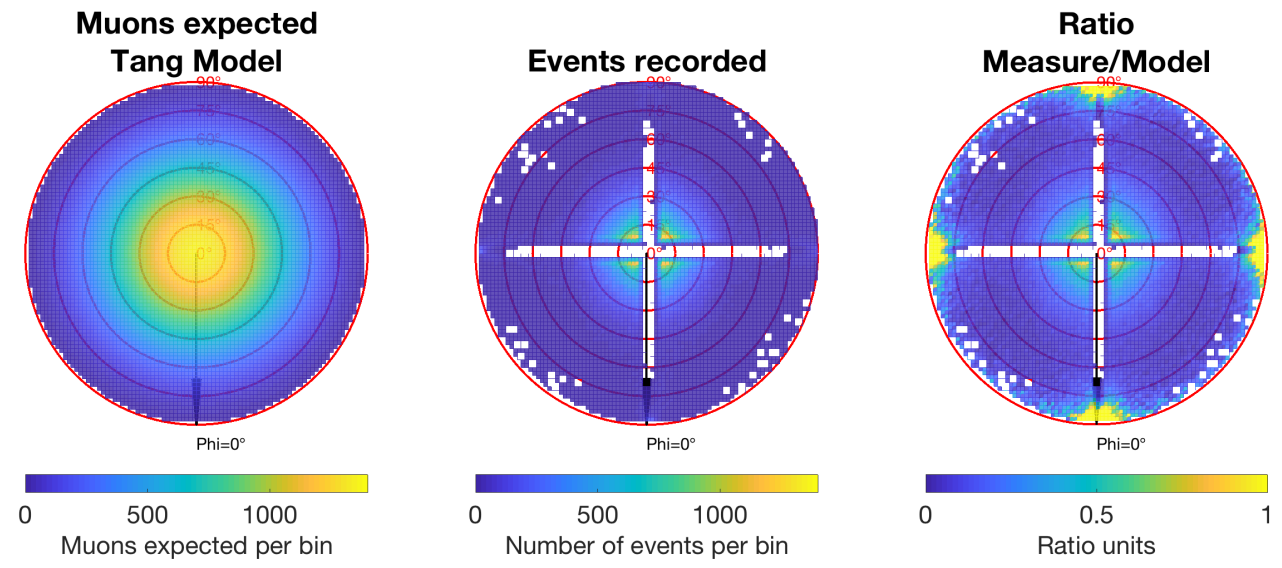

Fig. 2. (Left) Theoretical number of muons expected according to the Tang model, (Centre) Result of the open-sky acquisition and (Right) ratio between the recorded values and the expected ones.

The Figure 2 (Left) shows the theoretical muon arrival distribution for particles with energies over $105 \mathrm{MeV}$, integrated over $204 \mathrm{~min}$ (length of acquisition) to $0,52 \mathrm{~m}^{2}$ (active surface of detection). The central chart, Figure 2 (Centre), shows the successfully reconstructed events.

The trajectory reconstruction algorithm is nowadays unable to track muons with angles close to the orthogonal axis of the detection plane (up to $\sim 10^{\circ}$ ), or with trajectories superimposed to the $\mathrm{X}$ and $\mathrm{Y}$ micromegas readout tracks. This limitation is a combination of the hard limit imposed by the hardware design due to the TPC height $(5 \mathrm{~cm})$ and readout tracks pitch $(1 \mathrm{~mm})$ and the reconstruction algorithm, which requires well fitted projections to calculate the muon passage trajectories. The reconstruction artefact in the zones with $\theta \in$ $[80,90]$ and $\varphi$ near $0,90,180$ and 270 degrees is a side effect of the reconstruction algorithm. This zone, recognisable in yellow in the Figure 1 (Right), should have no effect on the inversion results if the detector is correctly positioned towards the target.

Lastly, Figure 2 (Right) can be interpreted as an efficiency distribution of the muon trajectory reconstruction and it can be used to correct the muon distribution with imaging purposes.

\section{Experimental results}

The first acquisition test under real field conditions aims at imaging a water reservoir in the village of Saint-Saturnin-les-Apt (Southeast of France). The dam structure is located at the lower point of a narrow valley overlooking the village. The following environment variables have been monitored: atmospheric pressure, precipitations, water level, air and water temperature, humidity. The terrain has been characterized with two different superimposed models corresponding to: (a) the water in the reservoir, its height is set to the water level at the moment of the beginning of the acquisition, and (b) the limestone of the valley and concrete/rock structure of the dam $\left(\rho=2.400 \mathrm{~kg} / \mathrm{m}^{3}\right.$ for all materials $)$. This model allows to calculate an opacity ${ }^{\dagger}$ chart from the point of view of the detector.

\footnotetext{
${ }^{\dagger}$ Medium opacity = sum of the distance travelled thought each material times its density, measured in meter water equivalent (mwe).
} 
Figure 3 shows the above-mentioned digital elevation model (DEM) and the location of the detector during the experience. The north axis is parallel to the $\mathrm{Y}$ axis of Figure 2 and corresponds to $\varphi=0$ in Figure 3 (a) and (b).

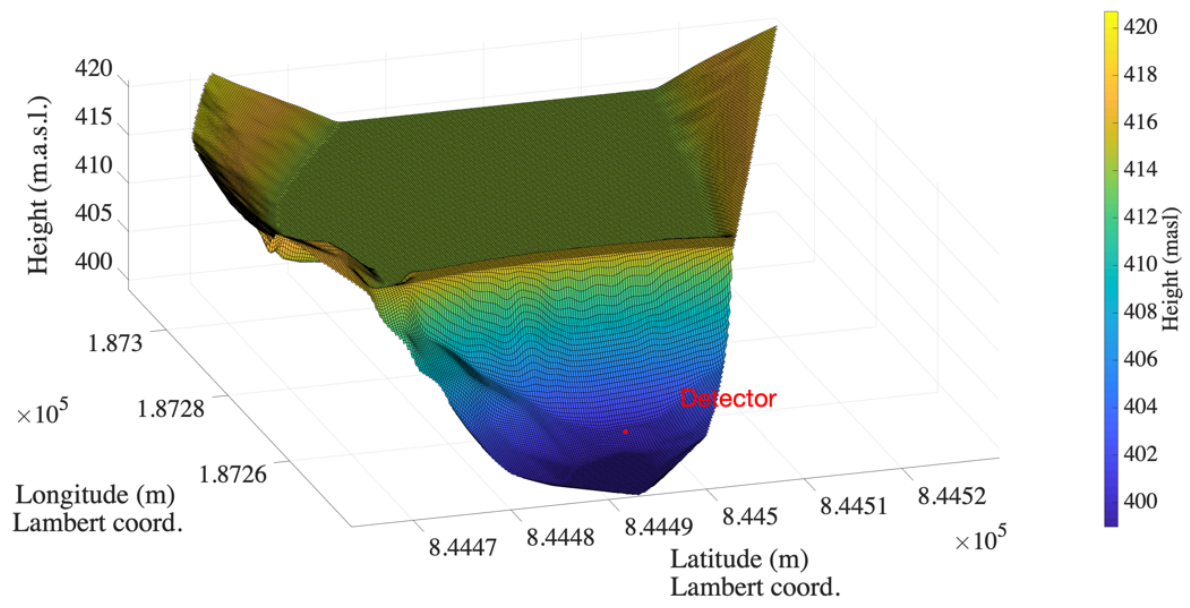

Fig. 3. Digital elevation model of the water reservoir and emplacement of the detector.

Figure 4 (a) has been calculated by means of the aforementioned DEM. The blank parts of the figure have three different explanations; (i) the muon flux attenuation is due to the atmosphere and is considered negligible at this level. (ii) The angular values exceed the limits of the DEM and the opacity is not calculated. (iii) The opacity exceeds the calculus cut-off ${ }^{\ddagger}$ value; high opacity values lead to severe muon flux attenuation. The Figure 4 (b) shows the experimental measurement of muon flux integrated over $\sim 90 \mathrm{~h}$. It is possible to distinguish the following elements: the red dotted line represents the position of the rock bottom of the dam and the right side stands for the cliff face in the east side of the reservoir. The green dotted line represents the position of the top of the dam. The zone (i) corresponding to the atmosphere presents an insignificant attenuation. On the contrary, the high values of attenuation in (iii) create data blanks in some points. As explained in Section 2, the blank near $\varphi=0^{\circ}$ and $\theta<10^{\circ}$ and the artefact near $\theta=80^{\circ}$ and $\varphi=0^{\circ}$ are due to the reconstruction algorithm.

\$The theoretical opacity calculated for zone (iii) doesn't reflect the real underground path of the particle due to the limited reach of the numerical model, hence the cut-off value. 

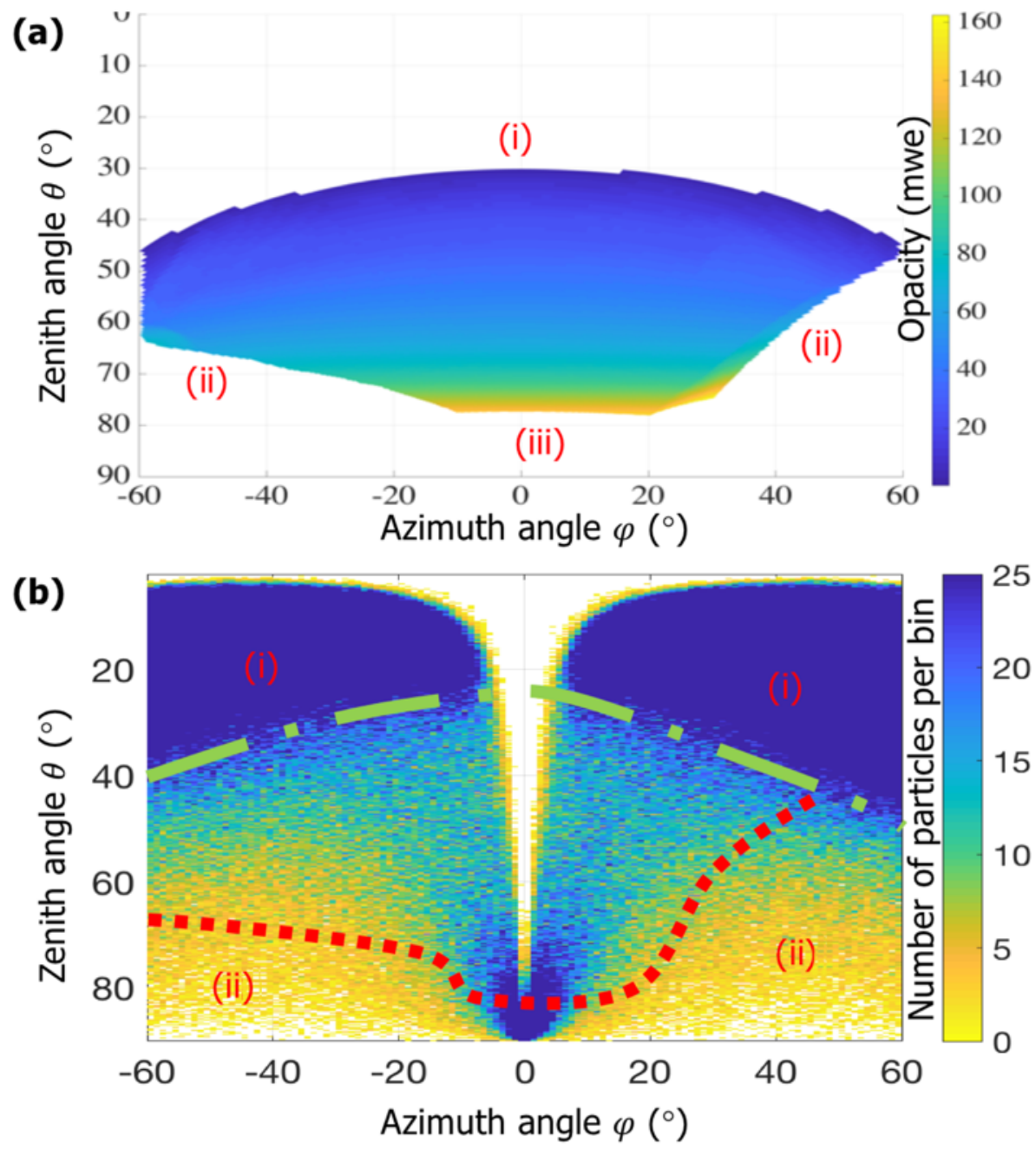

Fig. 4. (a) Simulation of the medium opacity. (b) Muon flux experimental measurement.

The experimental muon flux recorded is in good agreement with the opacity simulation and can be used to reduce the degrees of freedom when resolving the inverse problem in order to perform density TM. 
As for the temporal evolution of the data, the Figure 5 shows the variation of water level of the dam and muon flux. The value 0 of water level stands for the reference height of the water at the beginning of the experiment, 16 days before the data acquisition. On the other hand, the muon flux presented corresponds to the number of detected events per minute and per square meter of active surface. The flux is calculated considering all the logged events that contain the passage of a particle, regardless of the result of the subsequent track reconstruction.

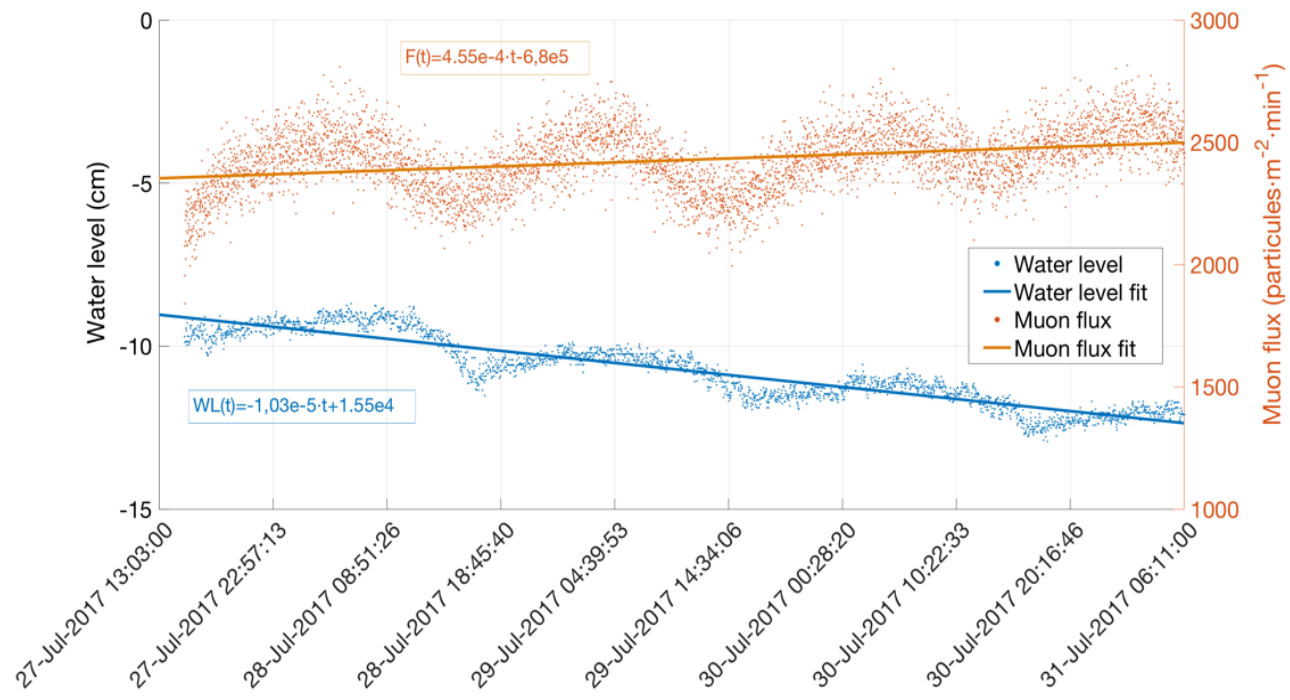

Fig. 5. Temporal evolution of (blue) water level of the dam and (orange) recorded muon flux.

In view of Figure 5, both series of data present a strong sine-wave behaviour. When compared individually against the environmental temperature during the data acquisition, they exhibit a strong inverse correlation. The value of Pearson's correlation coefficient for the water level and muon flux against temperature are $-0,62$ and $-0,22$ respectively, which indicates a non-lineal behaviour in the second case. The effect of the temperature in both the barometer used to determine the water level and the MUST ${ }^{2}$ detector, explains why when the measured water level increases locally, the measured muon flux increases as well contrary to what's expected. In normal conditions a water level rise should result in a flux drop and vice versa.

Nevertheless, when preforming a linear regression of the whole data, it is possible to appreciate that the emptying trend of the dam is translated into a rise of the muon flux. A Student's t-test has been performed between the initial and final halves of muon flux data series (corresponding to $45 \mathrm{~h}$ of data for each subseries). The $\mathrm{p}$-value $=1,77 \cdot 10^{-43}$ allows to reject the null hypothesis and quantifies the rise of the muon flux associated to the water level drop of $3,3 \mathrm{~cm}$ in $46,8 \pm 6,6$ particles per square meter and per minute (for a $95 \%$ confidence level).

\section{Conclusions}

In view of the results presented, the successful proof-of-concept trial allows to validate the MUST $^{2}$ camera for transmission muography purposes. During the campaign of measurements in the Saint-Saturnin-les-Apt dam, the field transportability and the capability 
to perform long-term out-of-lab measurements have been demonstrated. On the other hand, further data analysis development and experimental data is required to support the numerical model and resolve the inversion and obtain the medium density. Nowadays, a considerable number of detected events cannot be reconstructed, with the subsequent loss of information. The track reconstruction algorithm still has room for improvement in order to enhance the robustness of the results.

The authors thank the priceless support of the CERN's RD51 collaboration for the development of micro-pattern gas detectors technologies for all the assistance provided since the beginning of the project. We would like to highlight the kindness of the council of Saint-Saturnin-les-Apt for giving us access to the study site as well as the city archives. This research was performed within the framework of a CIFRE grant (ANRT contract \#2015/0325) and supported by the European Fund for Regional Development (FEDER).

\section{References}

1. L. Alvarez, et al. Search for hidden chambers in the pyramids, Science 167, 832-839 (1970).

2. K. Jourde, D. Gibert and J. Marteau. Improvement of density models of geological structures by fusion of gravity data and cosmic muon radiographies. Geosci. Instrum. Method. Data Syst., 4, 177-188. (2015).

3. K. Morishima et al. First demonstration of cosmic ray muon radiography of reactor cores with nuclear emulsions based on an automated high-speed scanning technology. 26th Workshop on Radiation Detectors and Their Uses in KEK, 27-36. (2012).

4. L. F. Thompson, et al. The application of muon tomography to carbon storage monitoring. 4th EAGE $\mathrm{CO}_{2}$ Geological Storage Workshop (2014).

5. T. K. Gaisser. Cosmic Rays and Particle Physics. New York, Cambridge university press edition. (1990).

6. A. Tang et al. Muon simulations for super-kamiokande, kamland, and chooz. Phys. Rev. D74, 053007 (2006).

7. N. Lesparre. Geophysical muon imaging: feasibility and limits. Geophys. J. Int. 183 1348 (2010)

8. I. Lázaro Roche. Design, construction and in situ testing of a muon camera for Earth science and civil engineering applications. Doctoral dissertation. https://hal.archivesouvertes.fr/tel-01895975 